Anæsthetics : Practical tuition for a fortnight or a month can be arranged. Limited to two post-graduates at a time.

Obstetrics: "Uterine Infections in Obstetrics and Gynæcology." A week's course of practical instruction is given during the 1 st and 3rd weeks of each month at St. Mary Abbots Hospital. Limited to four post-graduates at a time. Fee $\ell 15 \mathrm{~s}$. per week.

Clinical Assistantships: Two clinical assistants per calendar month (men only) will be appointed at the Lambeth Hospilal. Daily attendance 10-1 and 2-5. Fee $£ 55$ s.

Arrangements are made through the Fellowship.

A Guide Book, giving details of how to reach the various London Hospitals by tube, tram, or bus, can be obtained from the Fellowship. Price 6d. (Members and Associates, 3d.).

\title{
LOSS OF POWER IN THE HAND.
}

\section{By T. HOLMES SELLORS, M.A., M.CH., F.R.c.s.,} Assistant Surgeon, Queen Mary's Hosfital for the East End.

A WEAK grip or loss of strength in the hand is not uncommonly found as a sequel to some definite injury or lesion, and in many cases may constitute the first sign of abnormality in a more generalized disease. I have noticed that systematic examination and realization of the possible conclusions are not always satisfactory and consequently in this short article I propose to give an outline of examination and a classification of the diseases or conditions which may cause loss of power.

Examination.-Examination of the wrist and hand should always be undertaken systematically, comparing both sides all the time. The arms should be free of clothing as far as the shoulders and attention should be given to other parts of the body as may be necessary. First, have the hands palm upwards and look for any wasting, deformities or contractures, and obtain a general idea of the movements of the wrist and fingers. Then test the grip by making the patient grasp the crossed hands of the observer, and while this is being done the patient can be instructed to resist movements that should be attempted in all directions. A weakness in one particular direction affords a field for further study. Returning to the supine hands, movements can be amplified and the palm should be palpated for any thickening or swelling which may be present. It is always as well to be aware of the possibility of the presence of an early compound palmar ganglion which will be demonstrated by the presence of a cystic swelling above and below the anterior annular ligament.

Scars on the arm above the wrist require consideration according to their anatomical situation and the history of an old injury in a site such as round the elbow should draw attention to the possibility of nerve injury, of which the most usual is injury to the ulnar nerve at the internal condyle and less commonly to the median as a direct result of dislocation or fracture. In this connection the presence of a cubitus valgus, occurring after external condyle injuries more often than internal, may account for a tardy interstitial ulnar neuritis many years later. 
The hands may then be turned over and examined on the dorsal aspect and here any wasting of the intrinsic muscles is often peculiarly well shown, between the thumb and index finger in particular. A swelling over the extensor tendons is frequently due to a ganglion and these apparently simple conditions should not be ignored as a source of loss of power, especially in patients who depend for their livelihood on manual dexterity (e.g., pianists). The principal movement which has to be considered at this stage is wrist extension and the presence of wrist-drop may easily be overlooked if the hands are only examined in the supine position. Unilateral failure of the extensors entails a further investigation of the functions of the musculo-spiral nerve which may have been injured by pressure ("Saturday-night palsy," "crutch palsy"), or may have been interrupted by fractures of the shaft of the humerus or in an operation for osteomyelitis. Bilateral and incomplete wrist-drop should lead to the suspicion of lead neuritis, which would probably be accompanied by a blue line on gums and gastrointestinal symptoms.

To complete the general examination which should only take a few moments the reflexes should always be tested and compared and their absence or exaggeration noted : I emphasize this point because of a recent experience in which a man was examined by a series of people for loss of power of the hand. He suffered from a hemiplegia, admittedly with little spasticity of the arm, and almost every diagnosis that concerned the brachial plexus and nerve injuries was put forward in lieu of the correct one because the reflexes had not been tested; and this in face of the fact that there was no muscular wasting.

Sensory changes and trophic disturbances have not been commented on since their estimation is usually straightforward and the extent naturally varies with the type of lesion present. Examination must in this respect be made carefully and in addition to response to pin-prick and cotton-wool the question of any temperature loss is important in such cases as suspected syringomyelia.

Median Paralysis.-A rather more detailed consideration of the hand may be made to differentiate between the functions of the median and ulnar nerve. Take paralysis of the median first-the thenar eminence is flattened and the first interspace hollowed while the thumb is rotated into the plane of the rest of the hand, a feature which gives rise to the designation main de singe. It is not always realized that the human thumb is placed at right angles to the rest of the hand for all practical purposes and the only movement for which the median nerve can solely be responsible is that of abduction of the thumb; this is, as has been indicated, a movement away from the palm. In a similar connection it may be noted that the only other motor test for the median nerve depends on the integrity of flexor longus pollicis, which governs flexion of the terminal phalanx of the thumb. Consequently it is possible to overlook the motor loss occasioned by median nerve lesion unless examination is carried out with care. There is no need to comment in detail on the sensory loss and trophic disturbances over the radial three and a half digits.

Ulnar Paralysis.-Ulnar paralysis presents a more definite picture, and one which is much more easily recognized. The interosseous spaces show wasting and the hypothenar eminence becomes flattened and is associated with marked prominence of the metacarpal heads in the palm. The ulnar three fingers usually lie separated from each other, and in a position which later develops into a definite contracture consisting 
of hyperextension of the metacarpo-phalangeal joints with flexion of the interphalangeal articulations. If considered, this deformity is exactly the reverse of the "pen-holding" position induced by the action of normal interossei and lumbricales, and as the index finger is usually unaffected in ulnar palsy it earns the designation of the "pointing index." The power of abduction and adduction is lost, as may be shown by trying to make the patient open and close the extended fingers, and, if necessary, testing the grip obtained on a piece of paper placed between adjacent fingers. Adduction of the thumb also depends on the integrity of the ulnar nerve. Sensory and trophic loss over the ulnar one and a half fingers occurs on palmar and dorsal aspects unless the nerve has been injured below the origin of the dorsal cutaneous branch which leaves the main trunk 2 to 3 in. above the wrist-joint.

A combination of median and ulnar palsies produces a condition in which all the small muscles of the hand are paralysed with the result that all the fingers remain in a clawed position and the thumb cannot be opposed. This flat type of claw hand is known as the "main en griffe" and can be produced by a combined peripheral nerve injury or by any lesion which affects the first dorsal nerve in its motor part.

A short classification of the various forms of nerve lesion might be given at this stage, with a few suggestions as to how a diagnosis should be reached :-

Upper Motor Neurone Lesion.-Atrophy of muscle not great, spasticity, exagger. ated jerk reflexes.

Cortical and Pyramidal'Lesions.-Congenital spastic diplegia or hemiplegia-Little's disease. Dating from birth. Marked spastic contractures developed, arm flexed and prone, athetotic movements, subnormal mentality.

Acquired hemiplegia from cerebral hæmorrhage, thrombosis, trauma, \&c. History of onset and signs other than those in the arm make diagnosis more simple.

Disseminate Sclerosis.-Speech, gait, sphincteric loss of control, nystagmus. May include spasticity of part of upper limb.

Jacksonian Epilepsy.-History of attacks without loss of consciousness in definite order of spread. Weakness of affected parts may follow attack. Face and hand are usually associated.

Lower Motor Neurone Lesion.-Definite muscular atrophy.

Anterior Horn Cells.-Progressive muscular atrophy. Starts in hands, fibrillary twitchings, spread upwards in middle-aged men.

Amyotrophic lateral sclerosis. As progressive muscular atrophy, but with upper motor neurone lesion of legs leading to spasticity, \&c.

Acute anterior poliomyelitis. Rare in hand by itself, but may be present when whole upper extremity is affected. Acute febrile onset. Most common in children.

Syringomyelia. Affects posterior part of central canal of spinal cord often in cervical region. Hand paralysed early. Sensory changes important-touch unaltered loss of pain and temperature. Look for signs of scars (cigarette burns) or infection (Morvan's ulcers).

Intramedullary tumour of cord. Paresis and anæsthesia early. Pain not as severe as extra-medullary forms. Spasticity below.

Anterior Spinal Roots.-Injuries to spinal column. In dislocations of cervical, vertebræ roots on both sides may be damaged. Collapse of vertebra from Pott's disease 
not so likely to be associated with nerve changes as canal is relatively wide in this region.

Extra-medullary tumour. Loss of power and sensation. Pain severe and of root type, i.e., protopathic loss is greater than epicritic. Spasticity below.

Meningitis. Chronic hypertrophic form is seen in cervical region. Pain and wasting.

Ist Dorsal Root of Brachial Plexus.-Avulsion of root. Not common, usually just outside cord. Supraclavicular type of injury-strap-hangers palsy. Segmental type of motor and sensory loss if extra-spinal. In intra-spinal rupture hæmatomyelia or concussion of cord may result. Hæmatomyelia often has leg signs. Cervical sympathetic may also be injured, producing Horner's syndrome-Klumpke's palsy. Without sympathetic injury the condition may be called Aran-Duchenne type of paralysis which usually includes injury to $C 8$ as well.

Lowest Trunk of Brachial Plexus.-Cervical rib. True compression or friction syndrome. Symptoms more common on right. If prefixed plexus, condition is much more frequent. Abductor and opponens pollicis are affected first. Sympathetic changes due to inclusion of sympathetic fibres in nerve trunk. Vasomotor changespseudo-Raynaud's disease, pulse alteration, gangrene.

Cords of Brachial Plexus.-Dislocation of shoulder. Pressure of humeral head on inner cord in subcoracoid dislocation. Not common. Due to traction or direct thrust. Sensory loss ulnar border of forearm. Same mechanism will more commonly produce circumflex nerve injury with deltoid paralysis and sensory loss over the muscle: infraspinatus nerve is usually torn at the same time.

Fracture of the neck of humerus. Same remarks apply as to dislocation, but nerve may be more severely damaged by sharp ends of bone.

Median and Ulnar Nerves.-Injuries around elbow. Rare to find both nerves damaged together unless severe dislocation or fracture is present. Median most likely to be injured by forward dislocation of head of radius or badly displaced lower humerus fragments. Ulna more susceptible to direct injury or in fractures of internal condyle, also operative interference.

Injuries near wrist. Common. Hand pushed through plate-glass or wind-screen. Tendons damaged as well in most cases, watch for disability due to tendon section in addition to nerve. If tendons have to be explored see that nerves are intact if there is any doubt. Median can be damaged in forward dislocations of the semi-lunar bone and in attempts at reduction of this or of a Colles over a wedge. Stab wounds of palm and injudicious incisions in cases of infection should be remembered.

Neuritis. Arsenical neuritis affects arm in late stages of the condition. Skin pigmentation, keratosis, \&c. Alcoholic neuritis rarely affects arms; accompanied by sensory loss and severe pain. Toxic neuritis, including diphtheritic, occurs occasionally.

The discussion has so far been concerned with nerve lesions in so far as they affect the small muscles of the hand especially, and it has been shown that the first dorsal segment and its fibres passing into the median and ulnar nerves are the responsible agents. But there are other factors to be considered in weakness of the hand, and while still on the question of nervous origin another element can be mentioned. This is wrist-drop consequent on injury or neuritis of the musculo-spiral nerve. The loss of 
power is occasioned by inability to fix the hand in the "cock-up" position at which the optimum grip may be obtained.

To turn from nerves to other causes, one of the most obvious factors will be skeletal abnormalities. Bone deformity alters the mechanics of the wrist and hand and if they are beyond the limit of compensation weakness will ensue. The slight deformity that is seen as a late result in many cases of Colles' fracture is a case in point ; the radial abduction and loss of anterior concave bow at the lower end of radius may produce angle friction which reduces efficiency. Disease or injury of joints, among which one may instance tuberculosis of the wrist, rheumatoid arthritis and gonorrhœa are clearly detrimental to function.

Limitation of movement and fixation due to contracture of soft tissues and tendons can generally be recognized simply, but it is often difficult to assess the proportion of responsibility if both occur coincidentally. Careful examination of movements with the affected part in full flexion or extension may help if fixation of superficial tissues to ones deeper placed is studied. The presence of scars is suggestive of underlying damage, and burns, Dupuytren's contracture and congenital contractures should be borne in mind. One of the more important lesions connected with tendons is the presence of a chronic teno-synovitis which though occupational in many instances often turns out to be of a tuberculous nature. Compound palmar ganglion in the early stages is often difficult to recognize, as the anterior annular ligament checks any forward bulge over the wrist proper and leaves the cystic swelling to be palpated in the palm and in the lower forearm. In more advanced cases the ganglion is obvious and melonseed bodies may be felt within it. In these cases it is always advisable to examine the fingers for scars and to inquire into the occupation of the patient, for many cases of tuberculous infection occur in butchers, post-mortem and sanatorium attendants.

Still keeping to separate tissues as entities, muscle can be briefly discussed. Atrophy of disuse does not give much trouble in diagnosis if the history and comparative circumferences of the forearms are considered, and the only common lesion which may cause sudden loss of power by something "catching" the patient is a chronic strain of the nature of "tennis elbow." This shows a painful tender spot accurately localized over the external lateral ligament of the elbow-joint among the origin of the extensors, Myopathies avoid the hand and the pseudo-hypertrophic form is the only one which affects the forearm. The Landouzy-Dejerine and Erb's juvenile types confine their activities in the upper extremity to the region of the shoulder-girdle.

Weakness which has its origin in vascular causes can usually be detected with comparative ease though it may not always be so simple to elucidate the exact site of the vessel lesion. Volkmann's ischæmic contracture heralds its onset with acute pain and develops with distressing rapidity. The flexed phalanges can be straightened a little if the wrist is flexed, but extension of the wrist is not possible. The changes produced by cervical rib have already been touched on, and other conditions which may be mentioned are Raynaud's disease, aneurysm of the aorta and great vessels. Left-sided weakness is sometimes seen in a case of angina pectoris.

It has not been possible to deal with all the causes of loss of power in a brief survey, but I would like to emphasize the necessity for a broad outlook in the consideration of a problem of this nature. It is quite common to see an examiner surgically minded intent on proving that the condition is produced by a brachial plexus or nerve 
trunk injury without sufficient clinical evidence to support any of his conclusions : alternatively the physician may concentrate his energies on examination of the spinal cord and brain to the exclusion of other possibilities. A certain number of cases can be diagnosed simply and rapidly, but every now and then a case presents itself which offers considerable difficulty and can only be solved by a careful and detailed examination and deductions.

\title{
ELECTRICAL TREATMENT IN RHEUMATIC CONDITIONS.'
}

\author{
BY C. B. HEALD, C.B.E., M.D., M.R.C.P., \\ Physician in charge of Physical Medicine Department, Royal Free Hospital. Physician, British Red \\ Cross Clinic for Rheumatism. Consultant in Electro-therapeutics to the R.A.F.M.S.
}

Basic Diagnoses.-Lumbago, sciatica, rheumatism, yes, and even arthritis, are to my mind merely conventional names, and are in no sense diagnoses. Of this I can assure you, that if you employ these terms as diagnoses, and on them base your prescriptions for electrical treatments, many disappointing results will await you. I will go even further and state that because this has been done in the past, extensive discredit and disbelief in the value of electrical currents has been engendered.

Consider for a moment this situation. A patient comes into your consulting-room and complains of a pain or ache in the back; and, after listening to him and examining him, you tell him he has "lumbago," and he goes away and tells his friends the doctor has diagnosed his complaint, and he has got "lumbago." How ridiculous! If at the end of the consultation you were to say to this patient you have got an ache in your back, he would naturally be annoyed and tell you that that was what he told you on entering the room. But in actual fact that is all you have said, for the meaning of the word "lumbago" is pain in the loin.

Even rheumatism is derived from rheum, meaning the mucous discharge from the nostrils. If then we allow these names to lull us into a feeling of having made a diagnosis, we shall check progress towards real knowledge and continue to treat symptoms that may be, and usually are, only outlying manifestations of some general condition of the patient as a whole.

It will be asked at once whether I am prepared to substitute basic diagnoses for these symptomatic names. The answer is in the negative. For some years they must be retained as a temporary means of conventional communication between patient and doctor, and doctor and doctor. But there is a way in which a preliminary and honest mental break from these stupid so-called diagnoses may be made. If on our case sheets we write, Lumbago - - ? we have admitted to ourselves that lumbago is not a diagnosis ; we have reserved our judgment on the basic cause; we have obviously had the patient under consideration as a whole; and, above all, we have provided a continual stimulus to hunt for the cause.

If I write, Lumbago-cholecystitis, it is obvious that though diathermy may relieve and palliate the symptoms, it is our duty to inform the patient that the treatment is merely palliative and chancy and cannot be expected to cure him, and that other measures are required.

1 Delivered at the British Red Cross Clinic for Rheumatism, December 6, 1932. 